\title{
A boundary element method for the calculation of noise barrier insertion loss in the presence of atmospheric turbulence.
}

\author{
Yiu Wai Lam \\ University of Salford, Salford M5 4WT, UK. \\ Email: y.w.lam@salford.ac.uk
}

\begin{abstract}
Atmospheric turbulence is an important factor that limits the amount of attenuation a barrier can provide in the outdoor environment. It is therefore important to develop a reliable method to predict its effect on barrier performance. The boundary element method (BEM) has been shown to be a very effective technique for predicting barrier insertion loss in the absence of turbulence. This paper develops a simple and efficient modification of the BEM formulation to predict the insertion loss of a barrier in the presence of atmospheric turbulence. The modification is based on two alternative methods: 1) random realisations of log-amplitude and phase fluctuations of boundary sources; and 2) de-correlation of source coherence using the mutual coherence function (MCF). An investigation into the behaviours of these two methods is carried out and simplified forms of the methods developed. Some systematic differences between the predictions from the methods are found. When incorporated into the BEM formulation, the method of random realisations using only phase fluctuations and the method of MCF de-correlation provides predictions that agree well with predictions by the Parabolic Equation method and by the Scattering Cross-Section method on a variety of thin barrier configurations. Surprisingly the turbulence effect predicted by random realisations of both log-amplitude and phase fluctuations are found to be significantly stronger than those
\end{abstract}


predicted by other methods even for cases well within the saturation limit of the amplitude fluctuation.

\section{Introduction}

Atmospheric turbulence is an important factor in outdoor sound propagation. It is particularly important for noise barriers since it can substantially reduce the insertion loss provided by a barrier [1]. Nowadays it is usual practice to place a limit on the maximum insertion loss of a single barrier to allow for possible turbulence effects. However the calculation of the exact effect of turbulence remains difficult. This is partly due to the difficulty in characterising atmospheric turbulence for acoustic propagation calculation as well as due to the lack of good prediction models that properly account for turbulence effect. Recently, with increased understanding of atmospheric physics and its influence on acoustic propagation, several efforts have been made to develop prediction models for turbulence affected barrier insertion loss. With a suitable turbulence model, the method of scattering cross-section (SCS) provides the most straightforward calculation [1,2]. Alternatively, the Parabolic Equation (PE) method, which is well developed for sound propagation in an inhomogeneous atmosphere, can be used for thin barriers by approximating the sound pressure on the barrier surface in the shadow side with zero sound pressure [3,4]. More recently, a substituted-source model (SSM) [5,6] has been proposed. In this model Rayleigh's integral is applied to replace the propagating wavefront above the barrier with a plane of point sources. Turbulence effect is then incorporated by the degradation of coherence between the sources using a mutual coherence function (MCF) [7,8]. With the exception of Reference [9], most of these efforts have been applied to thin barriers. 
In noise barrier design, it is known that the insertion loss of a wide barrier can be significantly higher than that of a thin barrier of the same height. There have been extensive works [10] on the optimisation of barrier insertion loss using different shapes. In these works, the boundary element method (BEM) [11] has been shown to be highly effective and accurate for predicting the insertion loss of barriers of complex shapes. BEM schemes that use special formulations of the Green's function to take into account of atmospheric refraction on sound propagation over a barrier have been proposed [12,13]. However almost all studies on complex shaped barriers do not consider the effect of turbulence. It remains uncertain as to how much of the theoretical improvements in the insertion loss of complex barrier can be translated into performance improvements in real outdoor environments. The problem is two fold. Firstly the presence of the barrier, especially one with a complex shape, can significantly alter the turbulence structure in the neighbourhood of the barrier. The prediction of this change would require numerical simulations using sophisticated techniques in computational fluid dynamics. Even if one can predict the changes, it would still be a difficult task to incorporate the changes into the isotropic, homogeneous turbulence model that is used in acoustics propagation calculations. It is of interest to note that the linearized Eulerian sound propagation model proposed recently by Blumrich and Heimann [14] can calculate the changes in the mean flow field due to an obstacle in the calculation of sound propagation, and may also offer a method for calculating the effect of a complex turbulence field in the formulation. The second problem is that there is little work looking at how to propagate the sound field from an arbitrary, complex barrier through a turbulence atmosphere. In theory the substitute-source model can be adapted for wide barriers by incorporating the no-turbulence diffraction of the wide barrier into the source strength distribution of the substituted sources on the source plane. The effect of the thickness of a barrier can also be accounted for in the scattering cross-section model by virtue of the limiting angles of the cross-section. However, 
since the boundary element method is such an effective method for the no-turbulence insertion loss of a complex shaped barrier, it would be very useful and convenient to be able to incorporate turbulence effects directly into the boundary element method.

Theoretically, turbulence effects can be accounted for in the BEM by deriving a Green's function that includes perturbations due to turbulence. Unfortunately a direct formulation of such a Green’s function usually requires an integral over a suitable representation of the perturbed region and the resulting model would be computationally intensive. In this paper we investigate an approximate method that will allow turbulence effects to be included in the BEM without a substantial increase in computation time. The method is based on a concept similar to the substitute-source model, in that the effect of turbulence can be represented by a degradation of coherence between sources, or by random fluctuations of the logarithmic amplitudes and phases of the sources. However, instead of creating an artificial source plane as in the substitute-source model, the boundary sources inherent in the BEM will be used. This allows a convenient integration of the calculation of diffraction and turbulence effects. Since the boundary in a BEM is always finite, this method also avoids the truncation of an infinite plane as is necessary in the substitute-source model. Another significant difference is that the boundary sources involved in a BEM model include both point and dipole sources.

To facilitate the development of this method, the first part of this paper will present an analysis of the effect of turbulence on multiple source radiation. An approximate solution that allows a quick estimation of highly uncorrelated turbulence fluctuations will be derived. It will also identify analytical and simulated differences between turbulence effect calculations using logarithmic amplitude and phase fluctuations and the mutual coherence function. The 
second part of the paper will then look at boundary sources on realistic thin barrier configurations and comparisons with results published in the literature.

\section{Turbulence Effect on Multiple Source Radiation}

\section{Random Realisations}

One way of representing the effect of turbulence on the total sound energy at a receiver radiated from multiple sources is to use the correlation function of the turbulence structure to generate random realisations of the log-amplitude and phase fluctuations of the source contributions. Averaging over a large number of these realisations will then give the expected value of the turbulence affected sound pressure at the receiver. Under this model the fluctuations of the contribution from a source to the receiver are given by a multiplication factor $e^{\alpha+j \beta}$, where $\alpha$ and $\beta$ are assumed to be Gaussian variables representing the fluctuations from the no-turbulence value of the log-amplitude and phase. Using a Gaussian turbulence model $[\mathbf{1 5}, \mathbf{1 6}]$, the correlation functions for the log-amplitude and phase fluctuations can be written as:

$B_{\alpha}(\rho)=\langle\alpha(\bar{r}) \alpha(\bar{r}+\bar{\rho})\rangle$

and

$B_{\beta}(\rho)=\langle\beta(\bar{r}) \beta(\bar{r}+\bar{\rho})\rangle$

where $<>$ means expected value, $\bar{r}$ the coordinate vector and $\bar{\rho}$ the transverse separation vector with magnitude $\rho$ from the coordinate $\bar{r}$. For a Gaussian turbulence model the correlation functions are given by,

$$
B_{\alpha}(\rho)=B_{\beta}(\rho)=b_{o} \frac{\Phi(\rho / \ell)}{(\rho / \ell)}
$$


where $b_{o}=\frac{\sqrt{\pi}}{2} \mu_{o}^{2} k^{2} L \ell$,

and $k$ is the wavenumber of the non-perturbed medium, $\mu_{o}$ and $\ell$ are the standard deviation of the fluctuating part of the index of refraction and the correlation length of the Gaussian spectrum, $L$ is the longitudinal distance between the sources and the receiver, and the function $\Phi(x)=\int_{0}^{x} e^{-u^{2}} d u$

Using the above correlation function, realisations of $\alpha$ and $\beta$ for each source can be obtained using the filtering procedure described in Reference [17]. Once obtained, the randomly realised log-amplitude and phase fluctuations for source $i$ can be written as:

$1+v_{i}=\left(e^{\alpha_{i}+j \beta_{i}}\right) e^{-b_{o}}$

where $v_{i}$ represents the linear fractional complex variation for source $i$ from the mean value, and the term $e^{-b_{o}}$ on the right hand side is a normalisation factor necessary to compensate for the change in the sound power due to the introduction of amplitude fluctuation [17]. Note that there is a typographical error in Reference [17] where $e^{-2 b_{o}}$ was mistakenly quoted as the normalisation factor.

With this notation, the turbulence affected sound pressure at the receiver from all the sources is then,

$$
p=\sum_{i=1}^{n} Q_{i}\left(1+v_{i}\right)
$$

where $n$ is the total number of sources, and $Q_{i}$ is the sound pressure contribution at the receiver from source $i$ in the absence of turbulence. The expected mean-square sound pressure $\left\langle|p|^{2}\right\rangle$ over a large number of realisations is then 


$$
\left\langle|p|^{2}\right\rangle=\left\langle\left|\sum_{i=1}^{n} Q_{i}\left(1+v_{i}\right)\right|^{2}\right\rangle=\left\langle\sum_{i=1}^{n}\left|Q_{i}\left(1+v_{i}\right)\right|^{2}+2 \sum_{i=1}^{n-1} \sum_{j=i+1}^{n} \operatorname{Re}\left(Q_{i}\left(1+v_{i}\right) Q_{j}{ }^{*}\left(1+v_{j}\right)^{*}\right)\right\rangle
$$

The expected mean-square sound pressure can be determined directly by numerical simulation. Here we wish to determine an estimate of $\left\langle|p|^{2}\right\rangle$ for a limiting case.

Since $Q_{i}$ are contributions without turbulence, and are therefore independent of the realisations, we can write,

$$
\left\langle|p|^{2}\right\rangle=\sum_{i=1}^{n}\left|Q_{i}\right|^{2}\left\langle\left|\left(1+v_{i}\right)\right|^{2}\right\rangle+2 \sum_{i=1}^{n-1} \sum_{j=i+1}^{n} \operatorname{Re}\left(Q_{i} Q_{j}^{*}\left\langle\left(1+v_{i}\right)\left(1+v_{j}\right)^{*}\right\rangle\right)
$$

If the turbulence fluctuations are small, i.e. $\alpha_{i}<<1$ and $\beta_{i}<<1$ which is true when $b_{o}<<1$, then the exponential terms on the right hand side of Equation (2) can be approximated to give, $1+v_{i} \cong 1+\alpha_{i}-b_{o}+j \beta_{i}$

Using this approximation, the terms in the first summation of Equation (5) can be written as $\left|Q_{i}\right|^{2}\left\langle\left(1+\alpha_{i}-b_{o}\right)^{2}+\beta_{i}{ }^{2}\right\rangle=\left|Q_{i}\right|^{2}\left(1+2\left(1-b_{o}\right)\left\langle\alpha_{i}\right\rangle-2 b_{o}+\left\langle\alpha_{i}{ }^{2}\right\rangle+b_{o}{ }^{2}+\left\langle\beta_{i}{ }^{2}\right\rangle\right)$

Since the Gaussian random variable $\alpha_{i}$ have zero mean, and the variance $\left\langle\alpha_{i}{ }^{2}\right\rangle$ and $\left\langle\beta_{i}{ }^{2}\right\rangle$ are both equal to $b_{o}$, the terms can be reduced to, $\left|Q_{i}\right|^{2}\left(1+b_{o}^{2}\right)$

The terms in the double summation in Equation (5) can be reduced in a similar way.

$$
\operatorname{Re}\left(Q_{i} Q_{j}^{*}\left\langle\left(1+v_{i}\right)\left(1+v_{j}\right)^{*}\right\rangle\right)=\operatorname{Re}\left(Q_{i} Q_{j}^{*}\left\langle\left(1+v_{i}+v_{j}+v_{i} v_{j}^{*}\right)\right\rangle\right)
$$

Using the approximation of Equation (6), and making use of the fact that the Gaussian random variables has zero means, this can be reduced to,

$$
\begin{aligned}
& \operatorname{Re}\left(Q_{i} Q_{j}{ }^{*}\left(1-2 b_{o}+b_{o}{ }^{2}+\left(1-b_{o}\right)\left(\left\langle\alpha_{i}\right\rangle+\left\langle\alpha_{j}\right\rangle\right)+\left\langle\alpha_{i} \alpha_{j}\right\rangle+\left\langle\beta_{i} \beta_{j}\right\rangle\right)\right) \\
& =\operatorname{Re}\left(Q_{i} Q_{j}{ }^{*}\left(1-2 b_{o}+b_{o}{ }^{2}+\left\langle\alpha_{i} \alpha_{j}\right\rangle+\left\langle\beta_{i} \beta_{j}\right\rangle\right)\right)
\end{aligned}
$$


The expected values of the cross coupling terms $\left\langle\alpha_{i} \alpha_{j}\right\rangle$ and $\left\langle\beta_{i} \beta_{j}\right\rangle$ will be zero if the variables are totally uncorrelated between sources $i$ and $j$. This is approximately true if the separation between the sources, $\rho_{i j}$ is much larger that the turbulence correlation length $\ell$. The validity of this assumption will be examined later using numerical simulations.

Under these assumptions Equation (5) can now be written as,

$$
\left\langle|p|^{2}\right\rangle=\sum_{i=1}^{n}\left|Q_{i}\right|^{2}\left(1+b_{o}{ }^{2}\right)+2 \sum_{i=1}^{n-1} \sum_{j=i+1}^{n} \operatorname{Re}\left(Q_{i} Q_{j}^{*}\left(1-2 b_{o}+b_{o}{ }^{2}\right)\right)
$$

Re-arranging gives,

$$
\left\langle|p|^{2}\right\rangle=\left[\sum_{i=1}^{n}\left|Q_{i}\right|^{2}+2 \sum_{i=1}^{n-1} \sum_{j=i+1}^{n} \operatorname{Re}\left(Q_{i} Q_{j}^{*}\right)\right]\left(1-2 b_{o}+b_{o}{ }^{2}\right)+2 b_{o} \sum_{i=1}^{n}\left|Q_{i}\right|^{2}
$$

Note that the square bracket in the first term on the right hand side is in fact the mean-square pressure in the absence of turbulence, $\left|p_{n t}\right|^{2}$,

$$
\left|p_{n t}\right|^{2}=\left[\sum_{i=1}^{n}\left|Q_{i}\right|^{2}+2 \sum_{i=1}^{n-1} \sum_{j=i+1}^{n} \operatorname{Re}\left(Q_{i} Q_{j}{ }^{*}\right)\right]
$$

and the summation in the second term on the right hand side is the incoherently summed mean square pressure, $\left|p_{\text {incoh }}\right|^{2}$,

$$
\left|p_{\text {incoh }}\right|^{2}=\sum_{i=1}^{n}\left|Q_{i}\right|^{2}
$$

The turbulence affected mean square sound pressure can then be written as,

$$
\left\langle|p|^{2}\right\rangle=\left(1-2 b_{o}+b_{o}{ }^{2}\right)\left|p_{n t}\right|^{2}+2 b_{o}\left|p_{\text {incoh }}\right|^{2}
$$

This formula can be easily evaluated since both $\left|p_{n t}\right|^{2}$ and $\left|p_{\text {incoh }}\right|^{2}$ are independent of turbulence and $b_{o}$ is a constant that can be calculated readily from Equation (1a). 
The influence of turbulence can be more conveniently examined by subtracting $\left|p_{n t}\right|^{2}$ from Equation (9) to give the expected net mean square sound pressure difference caused by turbulence, $d\left(\left|p_{\text {turb }}\right|^{2}\right)$, $d\left(\left|p_{\text {turb }}\right|^{2}\right)=\left\langle|p|^{2}\right\rangle-\left|p_{n t}\right|^{2}=\left.\left(-2 b_{o}+b_{o}^{2}\right) p_{n t}\right|^{2}+2 b_{o}\left|p_{\text {incoh }}\right|^{2}$

A typical case of interest is that the amplitudes of the sources are of similar order of magnitude so that no one source dominates all others. In this case when the source contributions are largely in phase (i.e. $\left|p_{n t}\right|^{2}>>\left|p_{\text {incoh }}\right|^{2}$ ), and $b_{o}$ is small, then the difference $d\left(\left|p_{\text {turb }}\right|^{2}\right)$ caused by turbulence is negative, meaning a reduction in sound pressure level as expected. The equation predicts the reduction to be mostly linearly proportional to $b_{o}$. On the other hand, when the source contributions are largely out of phase, then the difference is positive, meaning an increase in sound pressure level, again as expected. The increase is also predicted to be linearly proportional to $b_{o}$.

The accuracy of Equation (10) for sources with randomly generated phases was investigated by comparing its prediction of $d\left(\left|p_{\text {turb }}\right|^{2}\right)$ with that predicted by direct random realisations using Equation (4). 100 random realisations were used in each case to generate the expected value. The source separations were set to $1 / 5$ of a wavelength $(\lambda)$. Two frequencies, $500 \mathrm{~Hz}$ and $1000 \mathrm{~Hz}$ were used in the simulation. The separations were about $1 / 8$ to $1 / 16$ of the typical turbulence correlation length $\ell=1.1 \mathrm{~m}$ [16] of the Gaussian turbulence model used. This choice of source separations was made to test the error caused by assuming totally uncorrelated log-amplitude and phase fluctuations between sources in the derivation of Equation (10) in cases where the separation is much less than $\ell$. Furthermore $\lambda / 5$ is a commonly adopted criterion for discretisation in acoustic numerical methods such as the 
BEM. The turbulence strength was set to a typical value of $\mu_{o}=3 \times 10^{-6}$ [16]. The number of sources used ranges from 256 to 2048, which corresponds to physical sizes between approximately $35 \mathrm{~m}$ to $278 \mathrm{~m}$. The range of $b_{o}$ realised was between 0.0025 to 0.25 so that the small $b_{o}$ assumption of Equation (6) was satisfied. To condense the results, the calculated values of $d\left(\left|p_{\text {turb }}\right|^{2}\right)$ were normalised by the factor $b_{o}\left|p_{\text {incoh }}\right|^{2}$ and plotted in Figure 1. A linear regression of the scattered data is also shown in the Figure together with the ideal match line (i.e. the line for $y=x$ ).

Figure 1 shows that the trend followed by the randomly realised expected values is close to that predicted by the approximate formula of Equation (10), i.e. the linear regression line is close to the ideal match line. The scatter in the data is partly caused by the random nature of the realisations, and partly by the omission in Equation (10) of the correlation between the log-amplitude and phase fluctuations between nearby sources - the latter being more significant with smaller numbers of sources. An extreme example of the correlation effect can be seen by considering the case when source contributions from adjacent sources are completely out of phase. In this case cancellation occurs between each pair of adjacent sources. If the separation between adjacent sources is much smaller than the turbulence correlation length then the cancellation will not be significantly affected by turbulence. However since Equation (10) inherently assumes uncorrelated turbulence induced fluctuations, it will still predict a significant increase in sound level due to turbulence. Even with this possible error in mind, Equation (10) provides a good estimate of the turbulence effect over a large range of randomly generated phase patterns, as shown in Figure 1.

\section{Mutual Coherence Function (MCF)}


Although random realisations can simulate the turbulence effect directly, it requires a large number of realisations to minimise the random variation of the averaged value. An alternative and popular method to calculate the effect of turbulence on multiple source radiation is the use of a MCF to reduce coherence between each pair of sources. For a Gaussian turbulence model, the MCF is given by $[\mathbf{7 , 8}]$,

$\Gamma(\rho)=e^{-2 b_{o}\left(1-\frac{\phi(\rho / \ell)}{\rho / \ell}\right)}$

where the symbols are as defined above.

The mean square sound pressure at the receiver is then calculated from,

$$
|p|^{2}=\left[\sum_{i=1}^{n}\left|Q_{i}\right|^{2}+2 \sum_{i=1}^{n-1} \sum_{j=i+1}^{n}\left|Q_{i}\right|\left|Q_{j}\right| \cos \left(\theta_{i j}\right) \Gamma_{i j}\right]
$$

where $\theta_{i j}$ is the phase difference between $Q_{i}$ and $Q_{j}$, and the MCF $\Gamma_{i j}$ is calculated from Equation (11) with $\rho_{i j}$ being the transverse separation between sources $i$ and $j$. Since the MCF is defined by Equation (11), the sound pressure level calculated from Equation (12) is deterministic.

Equation (12) can be simplified by making an assumption similar to that used to derive Equation (10). If we assume that all the MCF $\Gamma_{i j}$ in Equation (12) can be approximated by the limiting value of the MCF when $\rho>>\ell$, then $\Gamma_{i j}=\Gamma_{\infty}=e^{-2 b_{o}}$ becomes a constant. This is similar to the assumption that the fluctuations between sources are uncorrelated. Again assuming that $b_{o}<<1$, then $\Gamma_{\infty} \cong 1-2 b_{o}$, and Equation (12) can be simplified to,

$$
|p|^{2}=\left[\sum_{i=1}^{n}\left|Q_{i}\right|^{2}+2 \sum_{i=1}^{n-1} \sum_{j=i+1}^{n}\left|Q_{i}\right|\left|Q_{j}\right| \cos \left(\theta_{i j}\right)\left(1-2 b_{o}\right)\right]=\left|p_{n t}\right|^{2}-2 b_{o}\left[2 \sum_{i=1}^{n-1} \sum_{j=i+1}^{n}\left|Q_{i}\right|\left|Q_{j}\right| \cos \left(\theta_{i j}\right)\right]
$$


The net mean square sound pressure difference caused by turbulence, $d\left(\left|p_{\text {turb }}\right|^{2}\right)$, can be calculated by subtracting $\left|p_{n t}\right|^{2}$ (Equation (7)) from Equation (12) or Equation (13)).

Equation (13) can be compared directly against Equation (9) in two simple cases. The first case is when the receiver is at a point of destructive interference at which $\left|p_{n t}\right|^{2}=0$. In this case the double summation in the last term of Equation (13) is equal in magnitude but opposite in sign to the incoherent sum $\left|p_{\text {incoh }}\right|^{2}=\sum_{i=1}^{n}\left|Q_{i}\right|^{2}$, and Equation (13) reduces to $|p|^{2}=2 b_{o}\left|p_{\text {incoh }}\right|^{2}$ at a destructive interference point. This is identical to that predicted by Equation (10) (or Equation (9)). The random realisation and MCF methods are compatible in this case.

The second case is when the receiver is at a point of complete constructive interference. Assuming that all the $Q_{i}$ has equal magnitude and phase, i.e. $Q_{i}=Q$ and $\theta_{i j}=0$, then the double summation in the last term of Equation (13) reduces to $n^{2}|Q|^{2}-n|Q|^{2}=\left|p_{n t}\right|^{2}-\left|p_{\text {incoh }}\right|^{2}$ and Equation (13) itself reduces to $|p|^{2}=\left(1-2 b_{o}\right)\left|p_{n t}\right|^{2}+2 b_{o}\left|p_{\text {incoh }}\right|^{2}$ at a constructive interference point. This only differs from that derived from random fluctuations (Equation (9)) by a $b_{o}^{2}\left|p_{n t}\right|^{2}$ term. Since it has been assumed in the derivation that $b_{o}<<1$, this difference should not be significant.

The above comparisons are based on simplified versions of the random realisation and MCF methods. Figure 1 has already shown that the simplified Equation (10) has good agreement with direct realisations. The agreement between the simplified MCF prediction, Equation (13), and the full MCF method, Equation (12), is even better, as can be seen in Figure 2, which uses the same simulation cases of Figure 1. The scatter of data is much less in Figure 2 
because of the deterministic nature of the MCF calculations. Remarkably, the assumption of $\Gamma_{\infty}$, even when the actual separations between adjacent sources are less than $1 / 8$ of the turbulence correlation length, produces very good agreement with that using the actual MCF. One should however remember that the phase distributions between sources in these simulated cases are randomly generated. As mentioned earlier under the discussion of Figure 1, a regular phase pattern may produce bigger discrepancies.

The above analysis has shown that the MCF method is compatible with the random realisation method in the two extreme cases where the contributions are either largely out-of-phase or inphase. The result for other phase distributions is shown in Figure 3 where the predictions from full random realisations are plotted together with those from the full MCF method against the

ratio of $\left|p_{n t}\right|^{2} /\left|p_{\text {incoh }}\right|^{2}$. The trends of the two sets of data are almost inseparable. This confirms that the MCF formulation is indeed compatible with the method of random realisations..

\section{Boundary Sources from Barrier Diffraction}

\section{Barrier Boundary Source Patterns}

The above analysis was carried out to examine the generic behaviour of the calculation models for turbulence effect. It was also an attempt to derive a simple model for quick estimation of the effect. To a large extent Equation (10) and Equation (13) are such models. Both can be calculated easily from the source description and both have been shown to agree very well with their corresponding full calculation methods over a large set of randomly simulated cases. Unfortunately the application of these simplified models to the calculation of 
turbulence affected barrier attenuation is limited due to two significant factors. First the barrier size is generally not very much larger than typical values of the Gaussian turbulence correlation length. This could limit the validity of the assumption of uncorrelated logamplitude and phase fluctuations between sources that are used in the derivation of Equation (10) and (13). It should however be noted that the typical values of Gaussian turbulence correlation length given in the literature are based on free field measurements and predictions within the boundary layer of the atmosphere near the ground. The presence of a complex barrier will change the turbulence structure and could potentially generate a wide spectrum of turbulence in which the equivalent value of $\ell$ could be significantly smaller than the free field values. Experimental data on this is still rare and should be a subject of further investigation. Secondly the boundary sources on the barrier surface are created by the incident sound pressure. Their magnitude and phase follows a regular pattern determined by the sourcebarrier configuration and the correlation between sources is expected to play a larger part in such situations. In order to determine the effect of regular source patterns on the approximations, numerical simulations were performed with the source contributions $Q_{i}$ calculated from real, 2-dimensional barrier configurations. The boundary sources on a thin barrier placed on a hard ground were calculated for a point source $100 \mathrm{~m}$ away and at ground level. The boundary sources were discretised using again the criterion of $\lambda / 5$ for source separation. The contributions from these boundary sources to receivers at the same height as the barrier top and along a horizontal range of $10 \mathrm{~m}$ to $1000 \mathrm{~m}$ from the barrier were calculated to give the $Q_{i}$.

Figure 4 shows the result for a $20 \mathrm{~m}$ high barrier at $50 \mathrm{~Hz}$. The $\mathrm{x}$-axis shows the values of $b_{o}$ at different horizontal distances. In this case $\lambda / 5$ is larger (about 1.24 times) than the turbulence correlation length $\ell$. The assumption of uncorrelated turbulence fluctuations between sources 
is met and the approximated models, Equations (10) and (13), agree closely with the corresponding full realisation and MCF models. The differences between the randomly realised and the MCF calculated values are small, as demonstrated earlier in Figures 3. The results from the two approximated models are indistinguishable within the thickness of the lines.

When the frequency is increased to $500 \mathrm{~Hz}$, the source separation $(\lambda / 5)$ becomes much smaller than the turbulence correlation length $\ell$. In this case large discrepancies appear between the approximate models and the full models, as shown in Figure 5 for the 20m barrier. Interestingly the approximate models still follow closely the trend of the variation as a function of $b_{o}$, although the magnitudes of the predicted differences are significantly different. The agreement is improved for a bigger boundary, as can be seen in Figure 6 when the height of the barrier is increased to $100 \mathrm{~m}$. However such a large barrier is unrealistic in practice. So it seems that the approximate models, although having good accuracy for randomly phased sources, only give good estimate of the turbulence effect on barriers when the discretised boundary sources are further apart than the turbulence correlation length $\ell$ (i.e. at low frequencies), or when the size of the barrier is much larger than $\ell$. In our simulations using a criterion of $\lambda / 5$, the former condition corresponds to frequencies below roughly $50 \mathrm{~Hz}$. At higher frequencies the approximate models can be used to provide a quick estimation of the upper limit of the effect of turbulence on barrier attenuation. For more accurate barrier calculations, either the full random realisation method or the full MCF method should be used.

\section{Formulation of Turbulence Effect in BEM}


The validity of applying the random realisation method or the MCF method to a boundary element model is, unfortunately, not certain. The MCF, Equation (11), and the correlation function, Equation (1), that are used to generate the random realisations are both derived for point sources, while in a BEM formulation, the boundary has both point and dipole sources. On a hard barrier, the surface velocity is zero and the radiation is purely from dipole sources in a boundary integral formulation (see Equation (14) below). Whether Equations (1) and (11) can be applied to these cases is not clear.

Another question concerning the application of the turbulence calculation methods to BEM is related to the formulation of the BEM itself. The formulation of the BEM for an exterior diffraction problem is written as a sum of the scattered pressure and the incident pressure. If one were to use an approximation (described later in more detail) of calculating the boundary sources, which give rise to the scattered pressure, by ignoring turbulence and then proceed to calculate the total pressure at the receiver as a sum of the scattered and incident pressure, then a question arises as to whether one should introduce turbulence fluctuations in both the incident and scattered pressures or just in the scattered pressure. This question is illustrated more clearly by the following consideration. If one considers the physical noise source as one source and the boundary of the barrier as just another source, then the attenuation behind the barrier is caused by the destructive interference of the contributions from these two sources. A large attenuation corresponds to the case when the contribution from the barrier source is of similar magnitude but opposite in phase to the incident sound pressure. If we apply the simplified models, either Equation (10) or Equation (13), which should be valid if the noise source is far (relative to $\ell$ ) from the barrier, then the expected increase in the mean square sound pressure caused by turbulence is of the order of $2 b_{o}$ times 2 times the square of the magnitude of the incident pressure $\left(\left|p_{\text {incoh }}\right|^{2}\right.$ being the incoherent sum of that of the incident 
and the scattered pressure, with both having similar magnitude). This would reduce the insertion loss to $0 \mathrm{~dB}$ even with a $b_{o}$ of 0.25 (about $250 \mathrm{~m}$ behind the barrier at $1 \mathrm{KHz}$, with $\mu_{o}$ $=3 \times 10^{-6}$ ), which seems unrealistic. One will therefore need to consider carefully how to incorporate the turbulence calculation methods into the BEM.

The basic BEM formulation for an exterior diffraction problem in a stationary atmosphere without turbulence may be written as [11]:

$$
\int_{s} p_{s}\left(r_{o}\right) \frac{\partial G\left(r \mid r_{o}\right)}{\partial n_{o}}-G\left(r \mid r_{o}\right) \frac{\partial p_{s}\left(r_{o}\right)}{\partial n_{o}} d s=\varepsilon p_{s}(r)
$$

where $p_{s}$ is the scattered pressure component and $G$ is the Green's function for the free space problem. $\boldsymbol{r}$ and $\boldsymbol{r}_{\boldsymbol{o}}$ are coordinate vectors where the subscript $o$ indicates a position on the surface of the barrier $S . \boldsymbol{n}_{\boldsymbol{o}}$ is the outward normal at $\boldsymbol{r}_{\boldsymbol{o}}$. The constant $\varepsilon$ takes the values of $1,1 / 2$, and 0 if $\boldsymbol{r}$ is respectively in the exterior region $V$, on the boundary $S$, or in the interior of the barrier $V_{\text {in }}$.

Equation (14) gives the scattered pressure $p_{s}$. The total pressure $p_{n t}$ at $\boldsymbol{r}$ is calculated from,

$$
p_{n t}(r)=p_{s}(r)+p_{\text {inc }}(r)
$$

where $p_{i n c}$ is the incidence pressure from the source. Since most physical problems are defined with boundary conditions specified for the total rather then the scattered pressure, Equation (14) needs to be modified. Firstly, one notes that,

$$
\int_{S} p_{\text {inc }}\left(r_{o}\right) \frac{\partial G\left(r \mid r_{o}\right)}{\partial n_{o}}-G\left(r \mid r_{o}\right) \frac{\partial p_{\text {inc }}\left(r_{o}\right)}{\partial n_{o}} d s=0,-\frac{1}{2} p_{\text {inc }}(r),-p_{\text {inc }}(r) \text { for } r \in V, S, V_{\text {in }}
$$

Adding Equations (14), (15) and (16) together results in the more common formulation for the total pressure $p_{n t}$ in $V$, 


$$
\int_{S} p_{n t}\left(r_{o}\right) \frac{\partial G\left(r \mid r_{o}\right)}{\partial n_{o}}-G\left(r \mid r_{o}\right) \frac{\partial p_{n t}\left(r_{o}\right)}{\partial n_{o}} d s+p_{i n c}(r)=\varepsilon p_{n t}(r)
$$

An exact solution of the BEM can be obtained if one can determine a Green's function for the turbulent medium, say for example by solving an integral over the turbulent domain. However this is likely to be rather computationally expensive.

The terms inside the integral may be considered as representing a distribution of point sources (involving $G$ ) and dipole sources (involving $\partial G$ ). Hence the simple concept of source decorrelation by turbulence could be applied to these sources. As a first approximation, the strength of these boundary sources may be determined by solving the integral equation (Equation(17) with $\boldsymbol{r}$ on the boundary $S$ ) for a stationary, non-turbulent medium. This is similar to the approximation used for the determination of the source strength in the substitute-source model of Reference [5], and should be acceptable if the actual source is close to the barrier so that the source-to-barrier propagation is not much affected by the turbulence.

Once the boundary sources are determined, the random realisation method can be used to generate log-amplitude and phase fluctuations on the sources, or MCF can be applied to decorrelate the source contributions. Representing the contribution from each of these sources by $Q_{i}$, then Equation (4) or Equation (12) can be used to determine the turbulence affected mean-square scattered pressure, from which the mean square sound pressure difference caused by turbulence, $d\left(\left|p_{\text {turb }}\right|^{2}\right)$, can be calculated.

Since the barrier attenuation is caused by destructive interference of the incident and the scattered sound pressure, a change in the scattered pressure will generally reduce the 
interference and hence the attenuation. Here we take the magnitude of the mean square difference, $d\left(\left|p_{\text {turb }}\right|^{2}\right)$, to represent the amount of energy scattered by turbulence, such that the mean-square total pressure with turbulence is calculated from,

$|p|^{2}=\left|p_{n t}\right|^{2}+\mid d\left(\left|p_{\text {turb }}\right|^{2}\right)$

where $\left|p_{n t}\right|^{2}$ is calculated from Equation (17) and $d\left(\left|p_{\text {turb }}\right|^{2}\right)$ is calculated separately from the boundary sources only. The reason for not applying the turbulence de-correlation directly to Equation (17) for the total pressure is that, in the current formulation, it is not appropriate to apply the turbulence effect to the direct incident pressure term, i.e. the $p_{\text {inc }}$ term in Equation (17). Physically there is no direct incident wave reaching a receiver in the shadow zone of a barrier, only diffracted wave reaches the shadow zone. The term is only an artefact of the BEM formulation - the physical sound pressure is calculated from the sum of the no-barrier incident wave and the radiation from the barrier boundary sources. The initial calculation of the boundary source distribution uses the no-turbulence formulation of Equation (17), i.e. the boundary sources are calculated from the no-turbulence incident pressure. The introduction of turbulence fluctuations in the incident pressure after the calculation of the boundary pressure will cause inconsistence in the BEM formulation. Since the calculation of the total pressure in the shadow zone in a BEM depends critically on the cancellation of the incident and the barrier scattered pressure, this inconsistence could result in an over-estimation of the turbulence effect. This inconsistence can be illustrated by considering a case deep in the shadow zone. Without turbulence the total pressure is small because of the barrier. In the BEM formulation the scattered pressure from the boundary sources is nearly the same amplitude but of opposite phase to the incidence pressure. The two pressure waves thus cancel out each other to leave a small total pressure. With very strong turbulence and large source/barrier separation, the phase coherence will be greatly reduced. If we apply the 
turbulence de-correlation to the incident wave as well as to the boundary sources then the two pressure waves will become incoherent. The resulting total sound energy will come from the sum of two equal amplitude but incoherent waves and will therefore be higher than the nobarrier incident energy, which is physically not feasible.

Note also that the absolute magnitude of $d\left(\left|p_{\text {turb }}\right|^{2}\right)$ is used in Equation (18). This assumes that the turbulence affected sound level will be higher than the no-turbulence level, and is therefore strictly speaking applicable to the shadow zone only.

The advantage of this approximate BEM formulation is that the calculation of the boundary surface pressure, which is usually the most time consuming part of the computation, is separated from the calculation of the turbulence effect. Furthermore, calculation of the contributions $Q_{i}$ needs to be done only once from the boundary integral for each receiver. Hence it is very efficient even for a large number of random realisations.

\section{Application to Thin Barriers in a Turbulent Atmosphere}

The above BEM formulation was applied to several of the 2-dimensional thin barrier configurations used in References [4], in which barriers of heights 10 and 20m were used and the source was placed $100 \mathrm{~m}$ away from the barrier. These were chosen so that the BEM predicted results can be compared with published predictions using the scattering crosssection method (SCS), the Parabolic Equation (PE) and by inference the substitute-source method (the SSM has been shown to agree closely with the PE in these cases [5]). In these configurations the barriers are all placed on an infinite hard ground. A Gaussian turbulence 
model is assumed with the parameters $\mu_{o}=3 \times 10^{-6}$ and $\ell=1.1 \mathrm{~m} .100$ realisations were used for all cases in the BEM with random realisations.

Placing the barrier on a hard ground presents another problem for the BEM/boundary source formulation. In a normal BEM calculation, the effect of the hard ground is modelled by generating mirror images of the source and the barrier. The mirror images are by nature perfectly correlated with the originals. When turbulence is introduced, the log-amplitude and phase fluctuations will also be mirrored by the hard ground. In the MCF formulation, the separation $\rho$ needs to be calculated from the physical reflected path rather than from the mirrored path. Generally this is not a problem but in cases where the source and/or receiver are also on the ground, both the geometry and the turbulence structure in the model become highly symmetrical and unexpected errors may occur, as can be seen in some of the Figures later on.

Figure 7 shows comparisons for a $10 \mathrm{~m}$ high barrier at $500 \mathrm{~Hz}$. The source is located on the ground at a horizontal distance of $100 \mathrm{~m}$ from the barrier. The Figure shows the sound pressure level relative to that in the absence of the barrier (labelled "Relative SPL" in Figure 7 and subsequent figures) at the height of the barrier along a horizontal range up to $1000 \mathrm{~m}$. The BEM predictions using different turbulence effect calculation methods are plotted together with the PE and scattering cross-section (SCS) predictions, from Reference [4]. The BEM with MCF prediction agrees fairly well with the PE and SCS predictions up to about $600 \mathrm{~m}$. At around $800 \mathrm{~m}$ there is an unexpected drop in the BEM with MCF predicted SPL before it recovers. This is due to the ground mirroring effect discussed above. To support this argument, additional calculations were performed for the same barrier configuration with the source height elevated to $0.5 \mathrm{~m}$ and $1 \mathrm{~m}$. The results are shown in Figure 8 . The elevation of 
the source from the ground to $1 \mathrm{~m}$ reduces the symmetry in the turbulence induced MCF pattern and the unexpected drop in the BEM with MCF calculated SPL at $800 \mathrm{~m}$ is completely eliminated.

In Figure 7, the BEM prediction using random realisations of both log-amplitude and phase fluctuations is similar to that by the MCF method over much of the lower frequency range. However the strong dip in the attenuation calculated by the MCF method at about $800 \mathrm{~m}$ is not seen in the result by random realisations. Instead a smaller dip is observed in the latter at around 600m. Overall it has better agreement with the PE and SCS predictions. This suggests that the random realisation method is less affected by ground symmetry than the MCF method, which relies on accurate calculation of the lateral path separations between sources. However in practical calculations, since the MCF calculation is deterministic and does not require averaging over a large number of realisations, it is a faster and more convenient method to use.

Also shown in the figure is the resulted calculated using the approximate MCF. The result for the approximate formula (Equation (9)) is not shown since it is almost identical to that of using the approximate MCF. Since the approximate MCF is not calculated from the actual path difference the result does not exhibit the dip at 800m shown in the full MCF result. However the overall effect of turbulence is over-predicted by about $5 \mathrm{~dB}$ at range above about 200m. It confirms that for a non-random phase distribution among the boundary sources, the approximate methods should only be considered as providing an upper limit estimate of the turbulence effect. 
Figure 9 shows the result for the same barrier and frequency but with the source 200m away. With the source further away one may expect larger errors due to the assumption of noturbulence in the calculation of the boundary sources in the BEM formulation. Interestingly this is not the case. The BEM prediction, using either MCF or random realisations with logamplitude and phase fluctuations, agrees extremely well with the SCS and PE predictions. The results are similar when the barrier height is increased to $20 \mathrm{~m}$, as can be seen in Figure 10. In this case the BEM with MCF prediction has a stronger interference pattern than expected. Again this is thought to be caused by mirroring effect with the source on the ground.

Finally Figure 11 shows the result for the same configuration as Figure 7 but at a higher frequency of $1 \mathrm{kHz}$. At this frequency the value of $b_{o}$ approaches 1 at $1 \mathrm{~km}$ range. Even with this high value of $b_{o}$ the BEM prediction still works well. The BEM predictions have fairly close agreement with the PE and SCS predictions, but with a stronger interference pattern. The BEM with both the approximated MCF predicts a very strong turbulence effect that reduces the relative sound pressure level to $0 \mathrm{~dB}$ at range greater than $600 \mathrm{~m}$.

Overall the comparisons show that the BEM with log-amplitude and phase fluctuations gives the best and most consistent agreement with predictions by SCS and PE. The BEM with MCF prediction has good agreement with the other methods overall but seems to be more strongly affected by the ground mirroring effect when the source is on the ground. These good agreements suggest that application of the turbulence correlation function (Equation (1)) and the MCF (Equation (11)) to dipole boundary sources is acceptable. It also shows that the method of random realisations and the method of MCF are indeed compatible in barrier calculations. 
The results presented here are mainly comparisons against other theoretical/numerical models. The validity of the methods will need to be checked against experimental results. This work is currently continuing and the findings will be presented in a later paper.

\section{Conclusions}

The methods of calculating turbulence effect by random realisations of log-amplitude and phase fluctuations and by mutual coherence function de-correlation were investigated. A simplified model based on the assumption of uncorrelated turbulence fluctuations between sources was developed for each of these methods. The two simplified models were shown to agree very well with their corresponding full models for radiations from multiple sources that have randomly generated phase distributions. When applied to barrier configurations where the boundary sources on the barrier have more regular amplitude and phase patterns, the simplified models were found to work well when the separation between the discretised sources (usually $\lambda / 5$ ) is equal to or larger than the Gaussian turbulence correlation length $\ell$, or when the boundary surface is very large compared to $\ell$. When these conditions are not met, the simplified models over-predict the turbulence effect and can only provide an upper limit estimation of the effect.

The analysis has also shown that the random realisation and the MCF methods of predicting turbulence effect are compatible when applied to the boundary source formulation.

The methods of random realisations and MCF de-correlations were applied to the boundary sources created by a BEM discretisation to develop a BEM formulation for the calculation of 
turbulence effect on the insertion loss of a barrier. Predictions from this BEM formulation were compared with published results predicted by the Parabolic Equation method and the Scattering Cross-section method on several thin barrier configurations. The BEM formulation with random realisations of log-amplitude and phase fluctuations was found to have good agreement with the other prediction methods. The BEM formulation with MCF de-correlation was also found to agree well with other methods but suffered more strongly from ground mirroring effect when the source is on the ground plane of a hard ground. These good agreements justify the application of the turbulence correlation function and the mutual coherence function that were derived for point sources to the boundary dipole sources in a BEM model. Further work will be required to check this finding against experimental results.

The developments presented in this paper are based on the Gaussian turbulence model. It is known that the Gaussian model is a simple but crude approximation of the turbulence spectrum and there are better representations such as the von Karman model and the Kolmogorov model [see for example References 6-8]. The MCF for a Kolmogorov model is known and can be applied instead of the Gaussian model MCF in the BEM formulation. Further work will be required to see if this can provide a better prediction in practice. Experimental data on the turbulence structure near a barrier are also badly needed to facilitate further developments in this area.

\section{Acknowledgements}

The author wishes to thank Dr. Jens Forssén of Chalmers University of Technology, Sweden for providing the prediction data of Reference [4] and for the helpful discussions that we had 
on this topic. This work is supported by the UK Engineering and Physical Science Research Council under grant number GR/M71459.

\section{References}

1. Daigle D. A., "Diffraction of sound by a noise barrier in the presence of atmospheric turbulence”, J. Acous. Soc. Am., Vol. 71, pp.847-854, 1982

2. Tatarskii V. I. The effects of the turbulent atmosphere on wave propagation. Keter Press. Jerusalem, 1971.

3. Gilbert K. E., Raspet R. and Di X., "Calculation of turbulence effects in an upwardrefracting atmosphere”, J. Acoust. Soc. Am., Vol. 87, pp. 2428-2437, 1990.

4. Forssén J., "Calculation of sound reduction by a screen in a turbulent atmosphere using the parabolic equation method”, Acustica-Acta Acustica, Vol. 84, pp. 599-606, 1998.

5. Forssén J., "Calculation of noise barrier performance in a turbulent atmosphere by using substitute-sources above the barrier”, Acustica-Acta Acustica, Vol. 86, pp. 269-275, 2000.

6. Forssén J., "Calculation of noise barrier performance in a three-dimensional turbulent atmosphere using the substitute-sources method”, Acustica-Acta Acustica, Vol. 88, pp. 181-189, 2002.

7. Ostashev V. E., Gerdes F., Mellert V. and Wandelt R., "Propagation of sound in a turbulent medium. II. Spherical waves”, J. Acoust. Soc. Am., Vol. 102, pp. 2571-2578, 1997.

8. Ostashev V. E. Acoustics in moving inhomogeneous media. E \& FN Spon (an imprint of Thomson Professional), London, 1997. 
9. Forssén J. and Ögren M, “Thick barrier noise-reduction in the presence of atmospheric turbulence: measurements and numerical modelling”, Applied Acoustics 63, pp.173-187, 2002.

10. Crombie D. H., Hothersall D. C., Chandler-Wilde S. N., “Multiple-edge noise barriers,” Appl. Acoust. Vol. 44 (4): 353-367, 1995.

11. Burton A. J. and Miller G. F., "The application of integral equation method to the numerical solution of some exterior boundary-value problems”. Proc. Royal Society of London, A323, pp.201-218, 1971.

12. Premat E., and Gabillet Y., “A new boundary-element method for predicting outdoor sound propagation and application to the case of a sound barrier in the presence of downward refraction”, J. Acoust. Soc. Am. 108 (6), pp. 2775-2783, 2000

13. Taherzadeh S., Li K. M., and Attenborough K., “A hybrid BIE/FFP scheme for predicting barrier efficiency outdoors”, J. Acoust. Soc. Am. 110 (2), pp.918-924, 2001

14. Blumrich R., and Heimann D., "A linearized Eulerian sound propagation model for studies of complex meteorological effects”, J. Acoust. Soc. Am. 112 (2), pp.446-455, 2002

15. Daigle G.A., Piercy J.E. and Embleton T.F.W., "Effects of Atmospheric Turbulence on the Interference of Sound Waves Near a Hard Boundary”, J. Acous. Soc. Am., Vol. 64, pp.622 - 630, 1978

16. Daigle G.A., Piercy J.E. and Embleton T.F.W., "Line-of-sight Propagation Through Atmospheric Turbulence Near the Ground”, J. Acous. Soc. Am., Vol. 74, pp.1505 - 1513, 1983

17. Forssén J., "Calculation of noise barrier performance in a turbulent atmosphere by using substitute sources with random amplitudes”, Proc. $9^{\text {th }}$ Int. Symp. On Long-Range Sound Propagation, The Hague, Netherlands, 2000. 


\section{Figure Captions:}

Figure 1. Correlation between turbulence effects calculated from full random realisations and from the approximate model of Equation (10). Randomly generated source phase patterns. $b_{o}$ up to 0.25 .

Figure 2. Correlation between turbulence effects calculated from the full MCF and from the simplified MCF. Randomly generated source phase patterns. $b_{o}$ up to 0.25 .

Figure 3. Comparison of turbulence effects calculated from random realisations (with both log-amplitude and phase fluctuations) and from MCF at difference settings of source patterns.

Figure 4. Comparison of turbulence effect predictions by the full and simplified methods for a $20 \mathrm{~m}$ high thin barrier at $50 \mathrm{~Hz}$. The separation between the discretised sources is 1.24 times the Gaussian turbulence correlation length.

Figure 5. Comparison of turbulence effect predictions by the full and simplified methods for a $20 \mathrm{~m}$ high thin barrier at $500 \mathrm{~Hz}$. The separation between the discretised sources is about $1 / 8$ of the Gaussian turbulence correlation length.

Figure 6. Comparison of turbulence effect predictions by the full and simplified methods for a $100 \mathrm{~m}$ high thin barrier at $500 \mathrm{~Hz}$. The barrier size is very large compared with the correlation length. 
Figure 7. Calculated relative SPL at $500 \mathrm{~Hz}$ as a function of horizontal range from a $10 \mathrm{~m}$ high thin barrier on a hard ground. The source is on the ground $100 \mathrm{~m}$ away from the barrier. The receiver height is the same as the barrier height. $\mu_{o}=3 \times 10^{-6}$ and $\ell=1.1 \mathrm{~m}$.

Figure 8. Effect of elevated source height on the BEM with MCF prediction of turbulence effect. Barrier configuration is the same as Figure 8.

Figure 9. Calculated relative SPL at $500 \mathrm{~Hz}$ as a function of horizontal range from a $10 \mathrm{~m}$ high thin barrier on a hard ground. The source is on the ground $200 \mathrm{~m}$ away from the barrier. The receiver height is the same as the barrier height. $\mu_{o}=3 \times 10^{-6}$ and $\ell=1.1 \mathrm{~m}$.

Figure 10. Calculated relative SPL at $500 \mathrm{~Hz}$ as a function of horizontal range from a $20 \mathrm{~m}$ high thin barrier on a hard ground. The source is on the ground $200 \mathrm{~m}$ away from the barrier. The receiver height is the same as the barrier height. $\mu_{o}=3 \times 10^{-6}$ and $\ell=1.1 \mathrm{~m}$.

Figure 11. Calculated relative SPL at $1 \mathrm{kHz}$ as a function of horizontal range from a $10 \mathrm{~m}$ high thin barrier on a hard ground. The source is on the ground $100 \mathrm{~m}$ away from the barrier. The receiver height is the same as the barrier height. $\mu_{o}=3 \times 10^{-6}$ and $\ell=1.1 \mathrm{~m}$. 


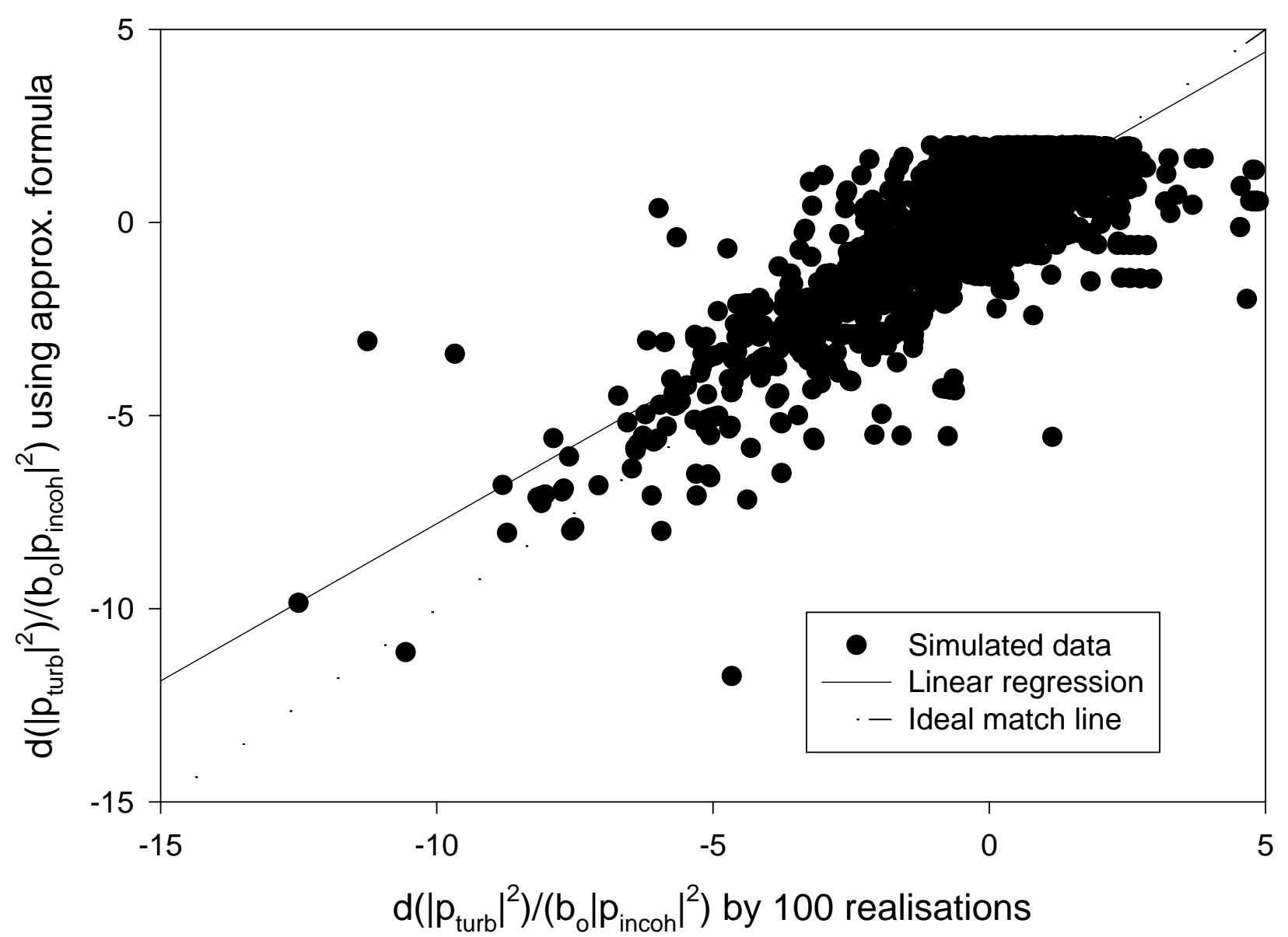

Figure 1 Correlation between turbulence effects calculated from full random realisations and from the approximate model of Equation (10). Randomly generated source phase patterns. $b_{o}$ up to 0.25 . 


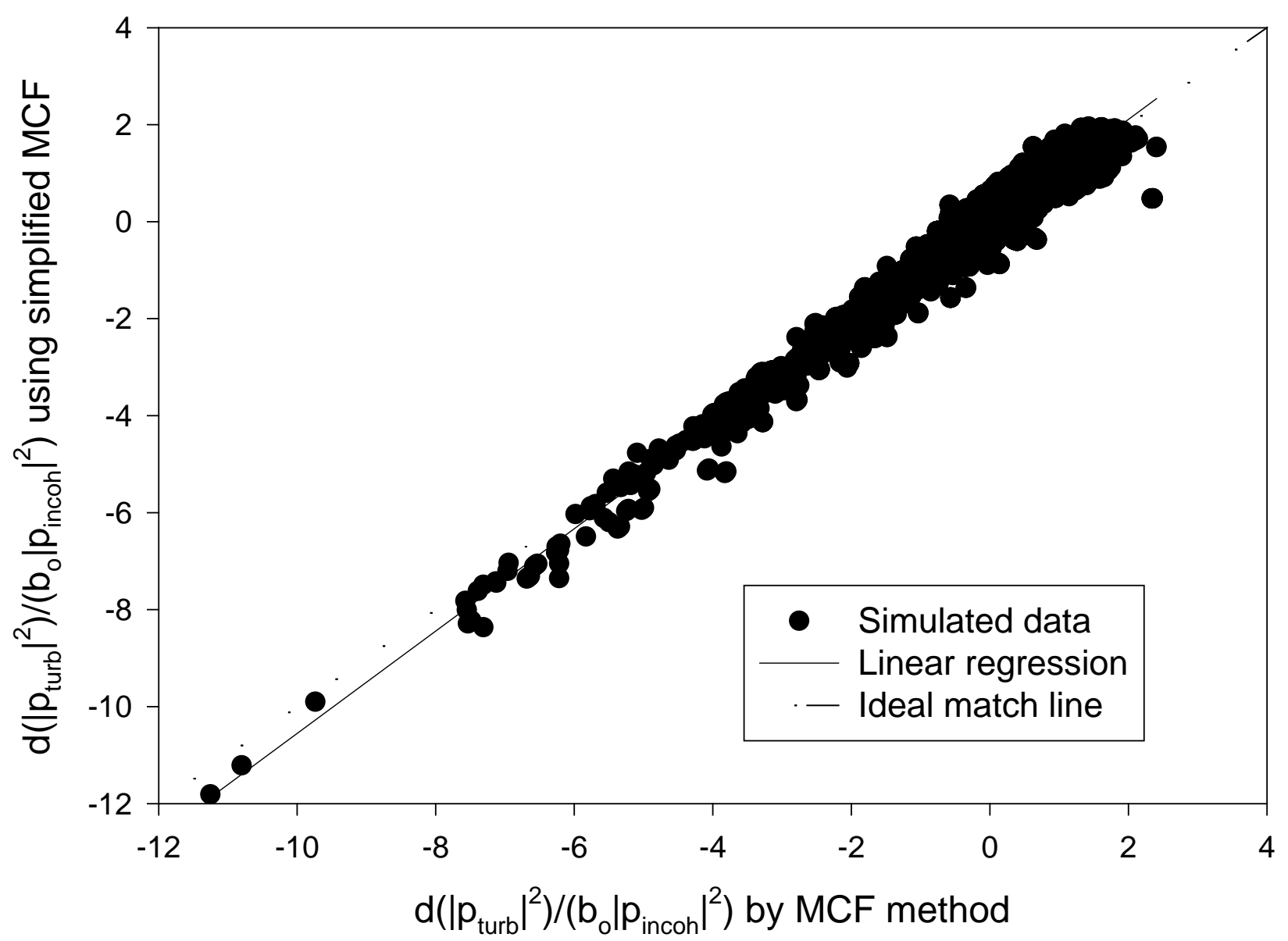

Figure 2 Correlation between turbulence effects calculated from the full MCF and from the simplified MCF. Randomly generated source phase patterns. $b_{o}$ up to 0.25 . 


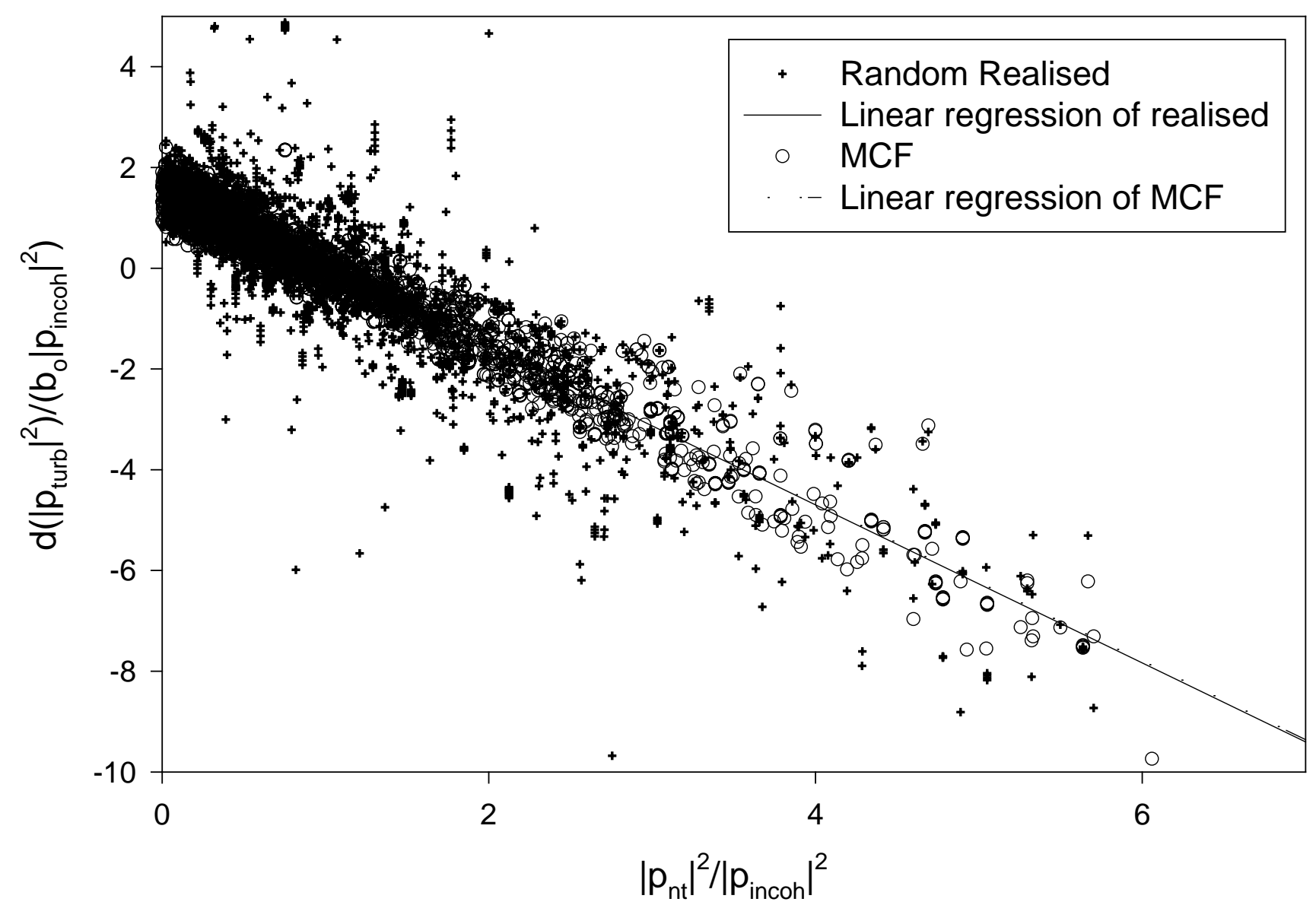

Figure 3 Comparison of turbulence effects calculated from random realisations (with both log-amplitude and phase fluctuations) and from MCF at difference settings of source patterns. 


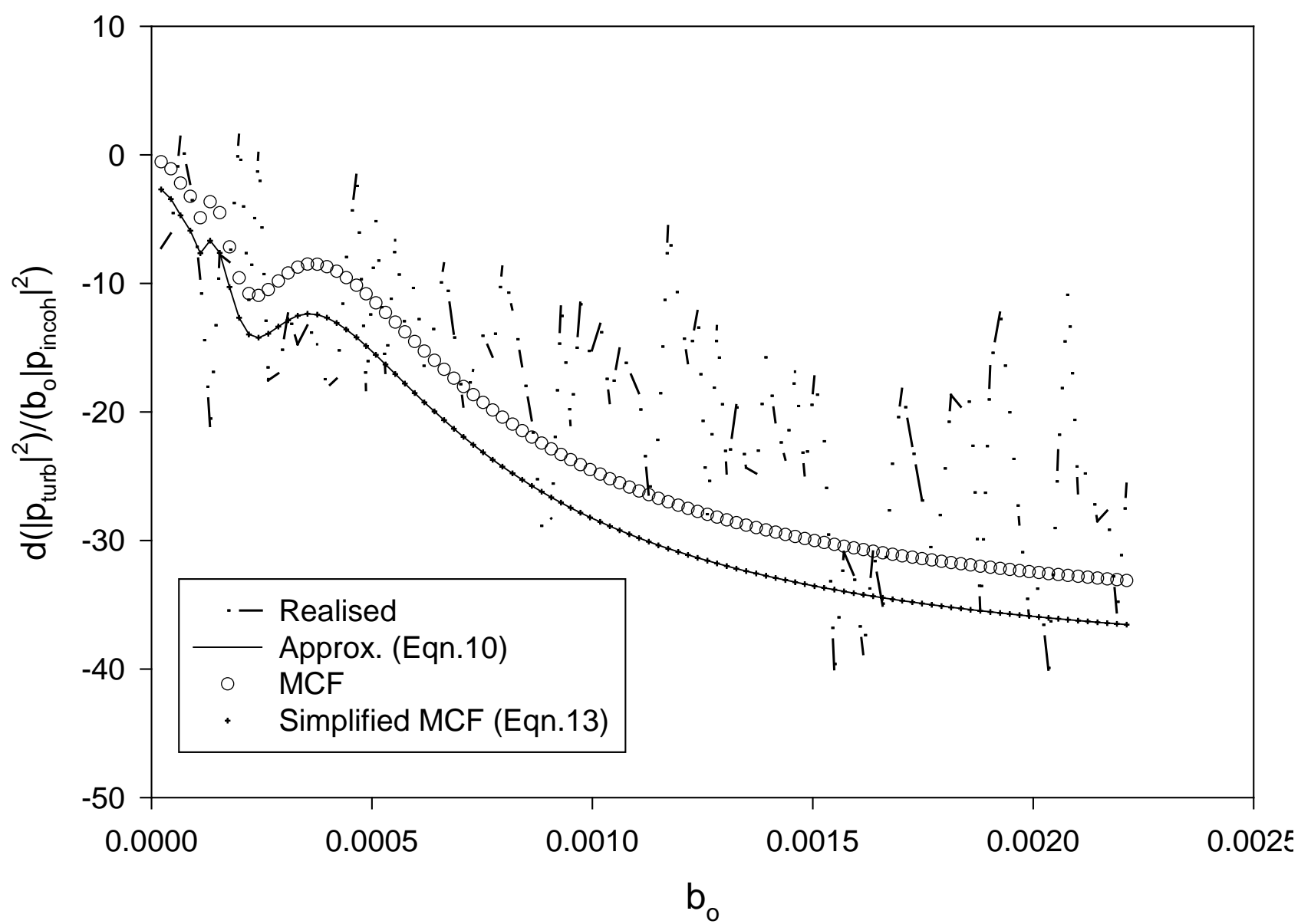

Figure 4 Comparison of turbulence effect predictions by the full and simplified methods for a $20 \mathrm{~m}$ high thin barrier at $50 \mathrm{~Hz}$. The separation between the discretised sources is 1.24 times the Gaussian turbulence correlation length. 


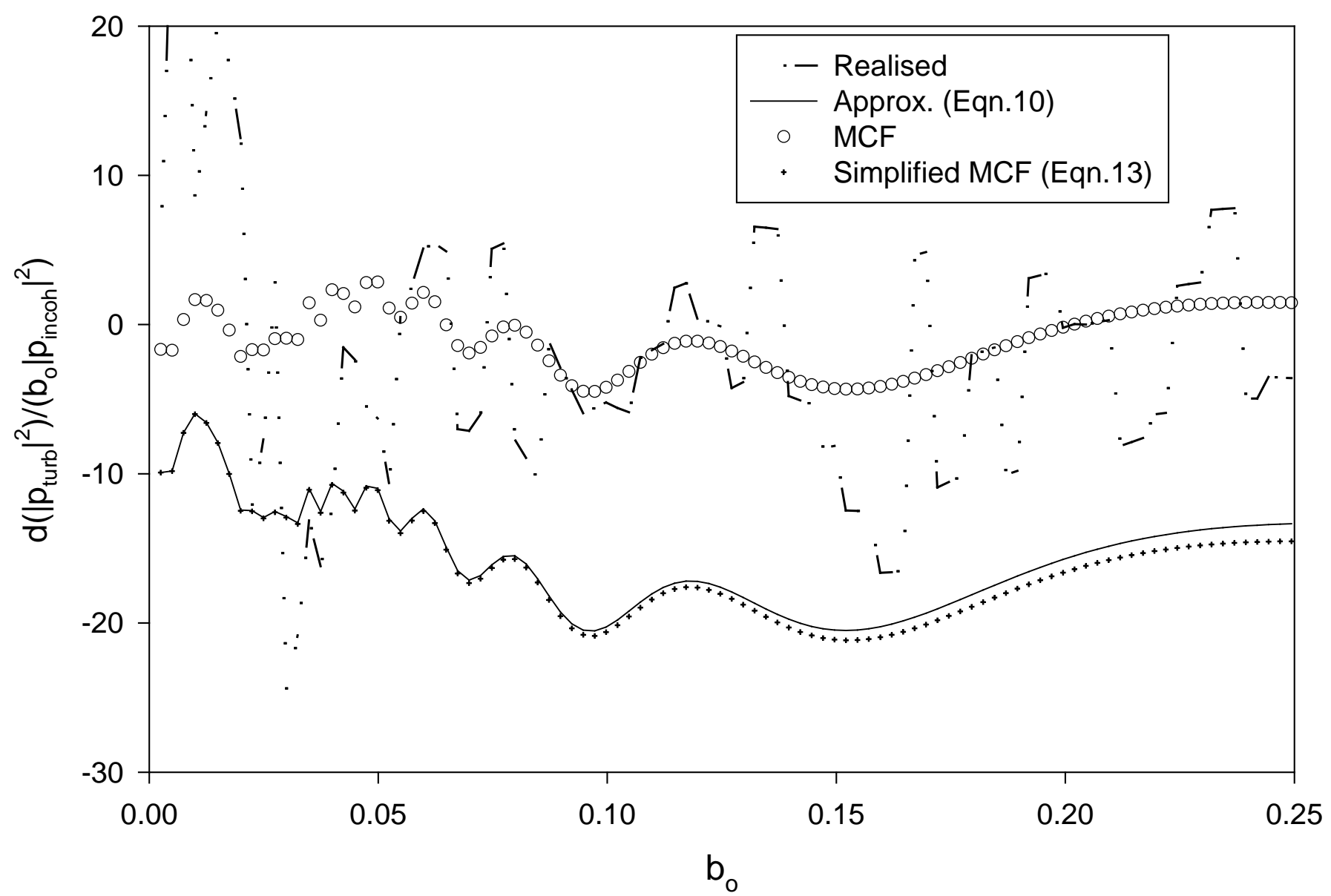

Figure 5 Comparison of turbulence effect predictions by the full and simplified methods for a $20 \mathrm{~m}$ high thin barrier at $500 \mathrm{~Hz}$. The separation between the discretised sources is about 1/8 of the Gaussian turbulence correlation length. 


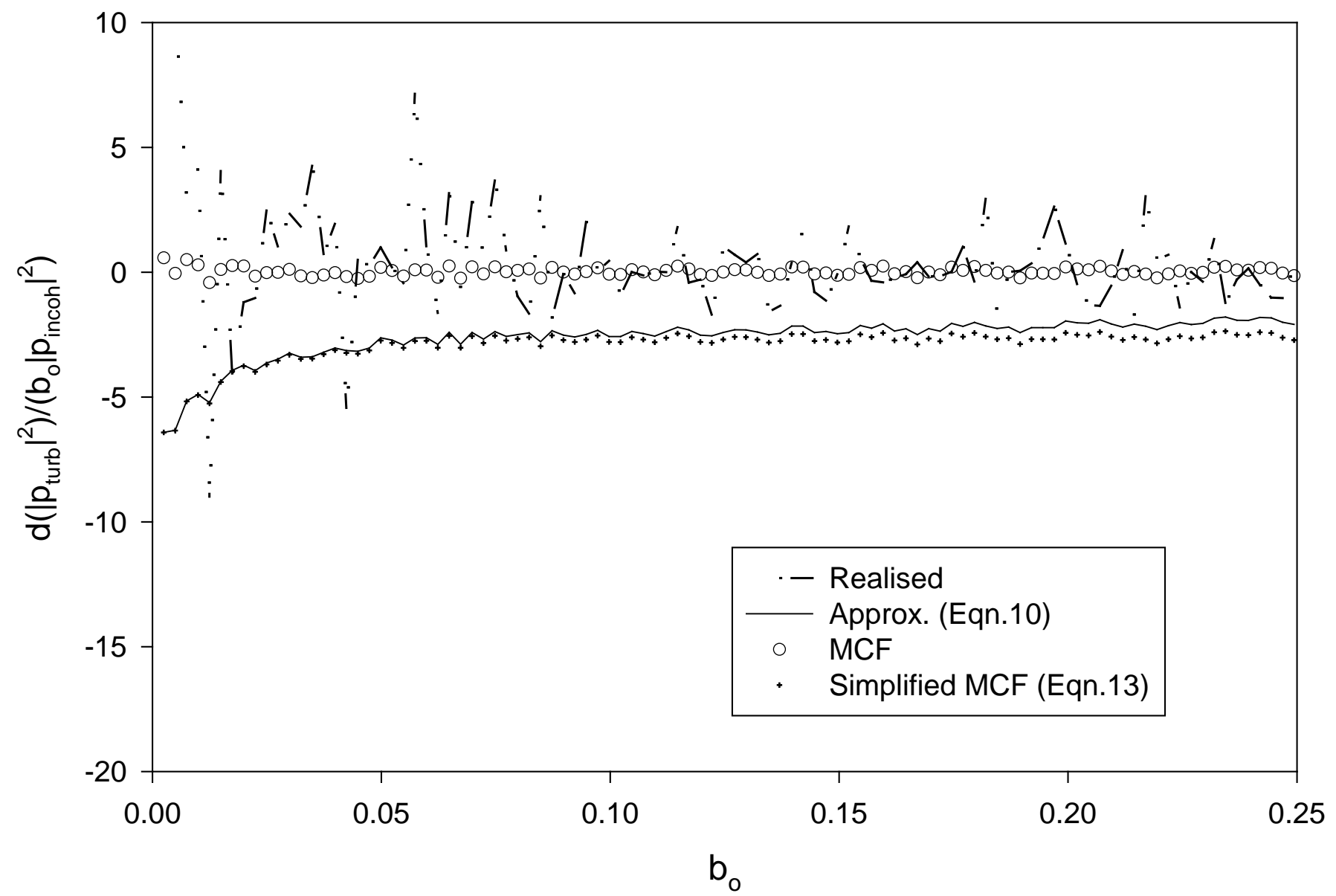

Figure 6 Comparison of turbulence effect predictions by the full and simplified methods for a $100 \mathrm{~m}$ high thin barrier at $500 \mathrm{~Hz}$. The barrier size is very large compared with the correlation length. 


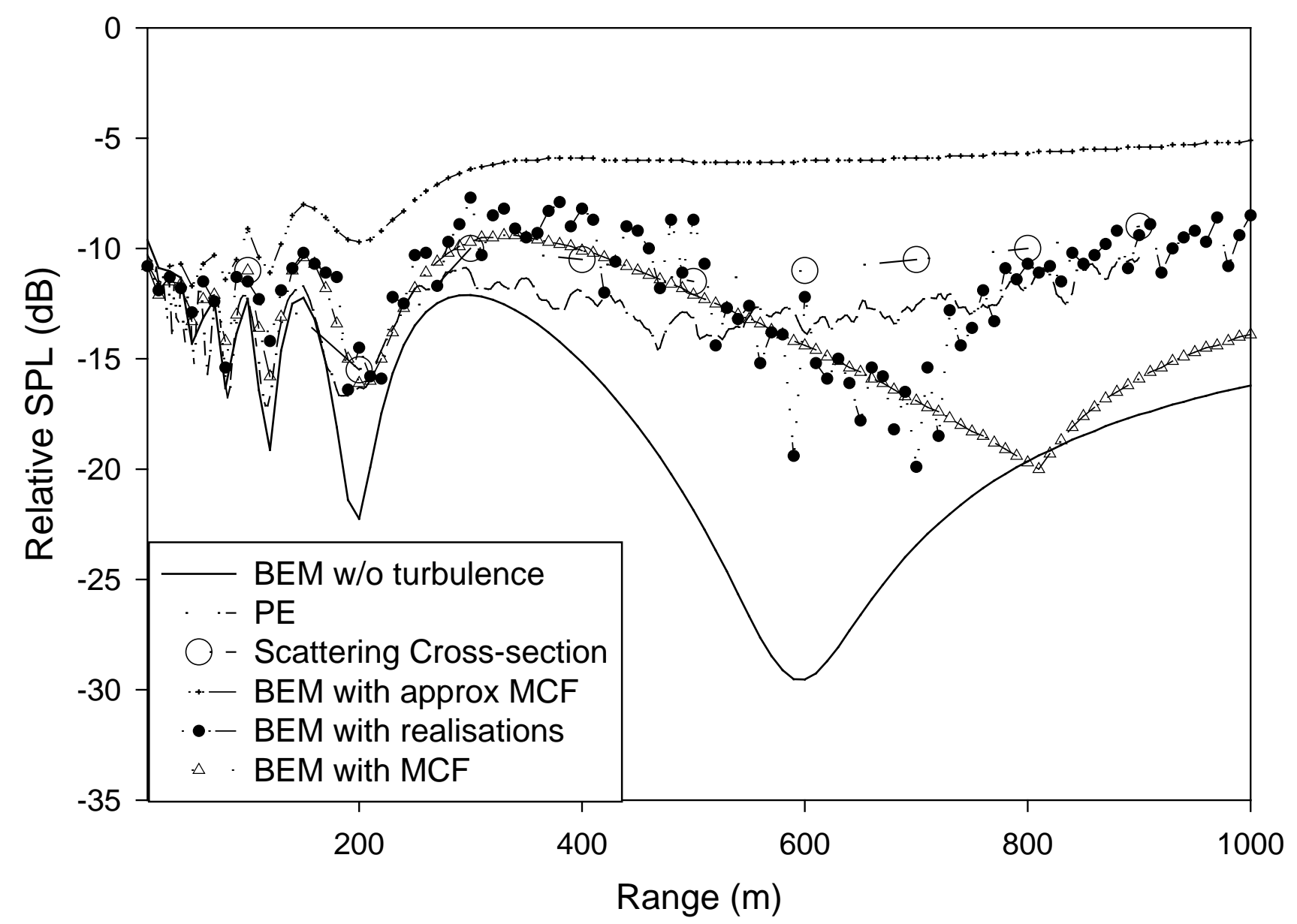

Figure 7 Calculated relative SPL at $500 \mathrm{~Hz}$ as a function of horizontal range from a $10 \mathrm{~m}$ high thin barrier on a hard ground. The source is on the ground $100 \mathrm{~m}$ away from the barrier. The receiver height is the same as the barrier height. $\mu_{o}=3 \times 10^{-6}$ and $\ell=1.1 \mathrm{~m}$. 


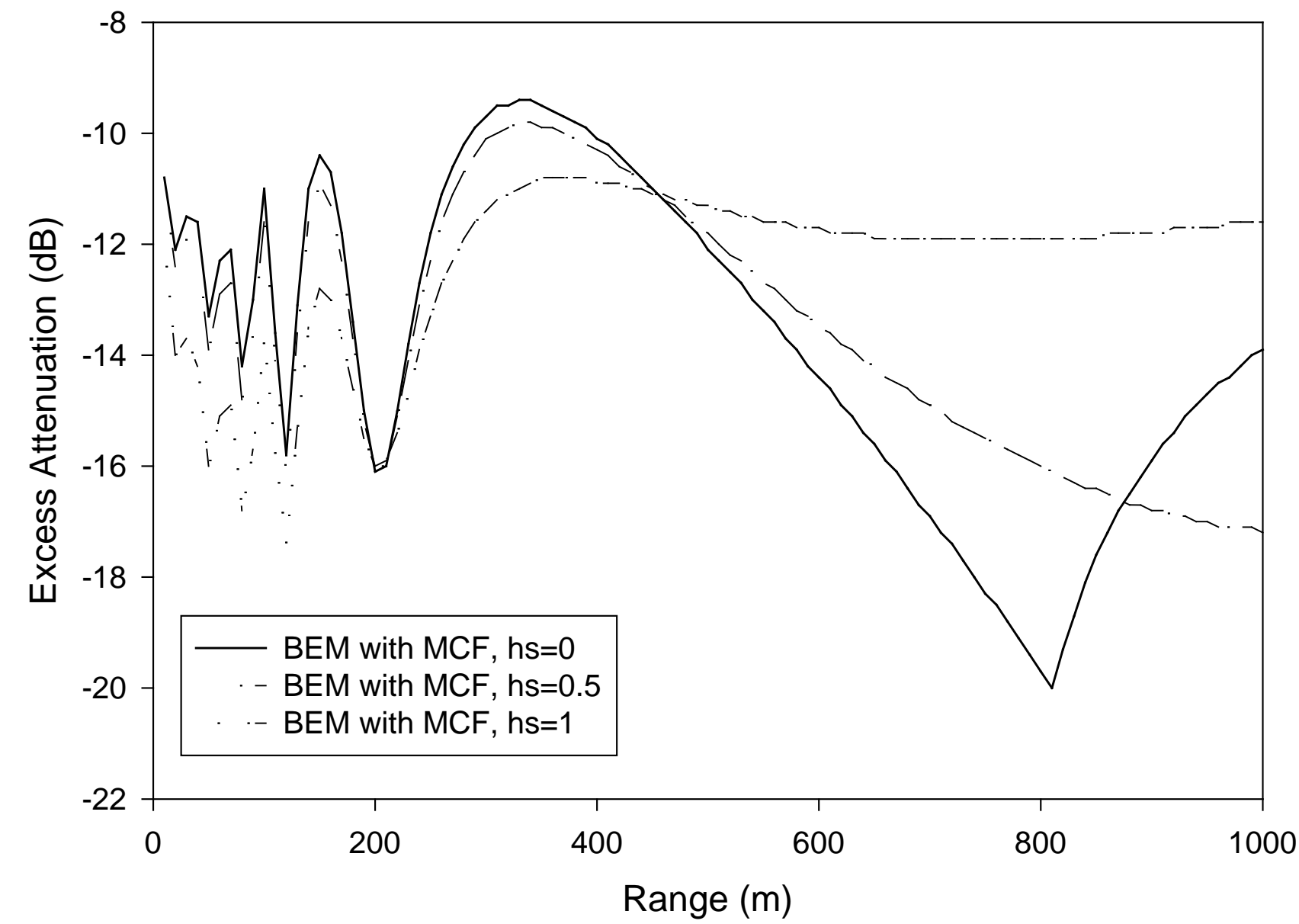

Figure 8 Effect of elevated source height on the BEM with MCF prediction of turbulence effect. Barrier configuration is the same as Figure 8. 


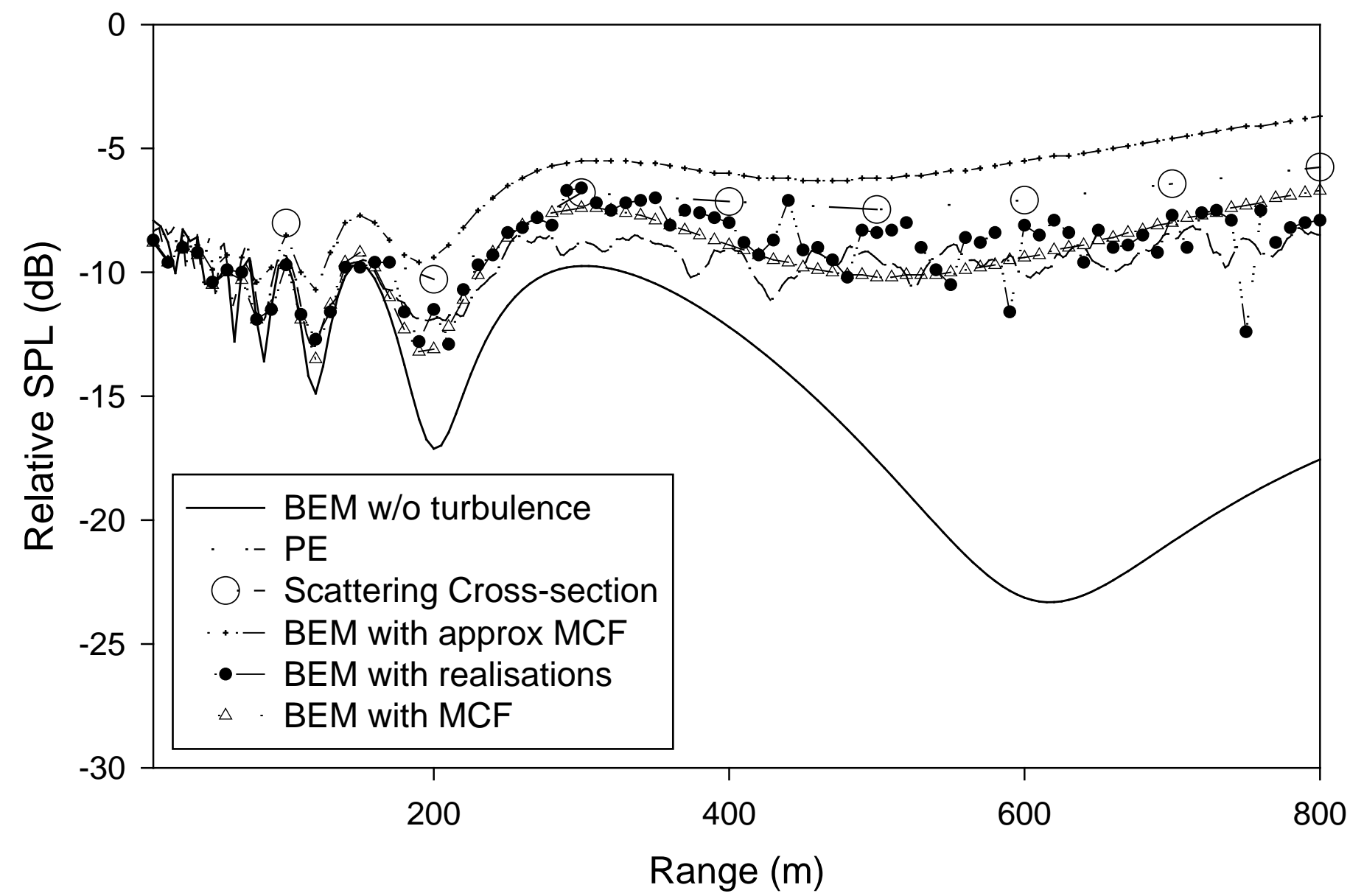

Figure 9 Calculated relative SPL at $500 \mathrm{~Hz}$ as a function of horizontal range from a $10 \mathrm{~m}$ high thin barrier on a hard ground. The source is on the ground $200 \mathrm{~m}$ away from the barrier. The receiver height is the same as the barrier height. $\mu_{o}=3 \times 10^{-6}$ and $\ell=1.1 \mathrm{~m}$. 


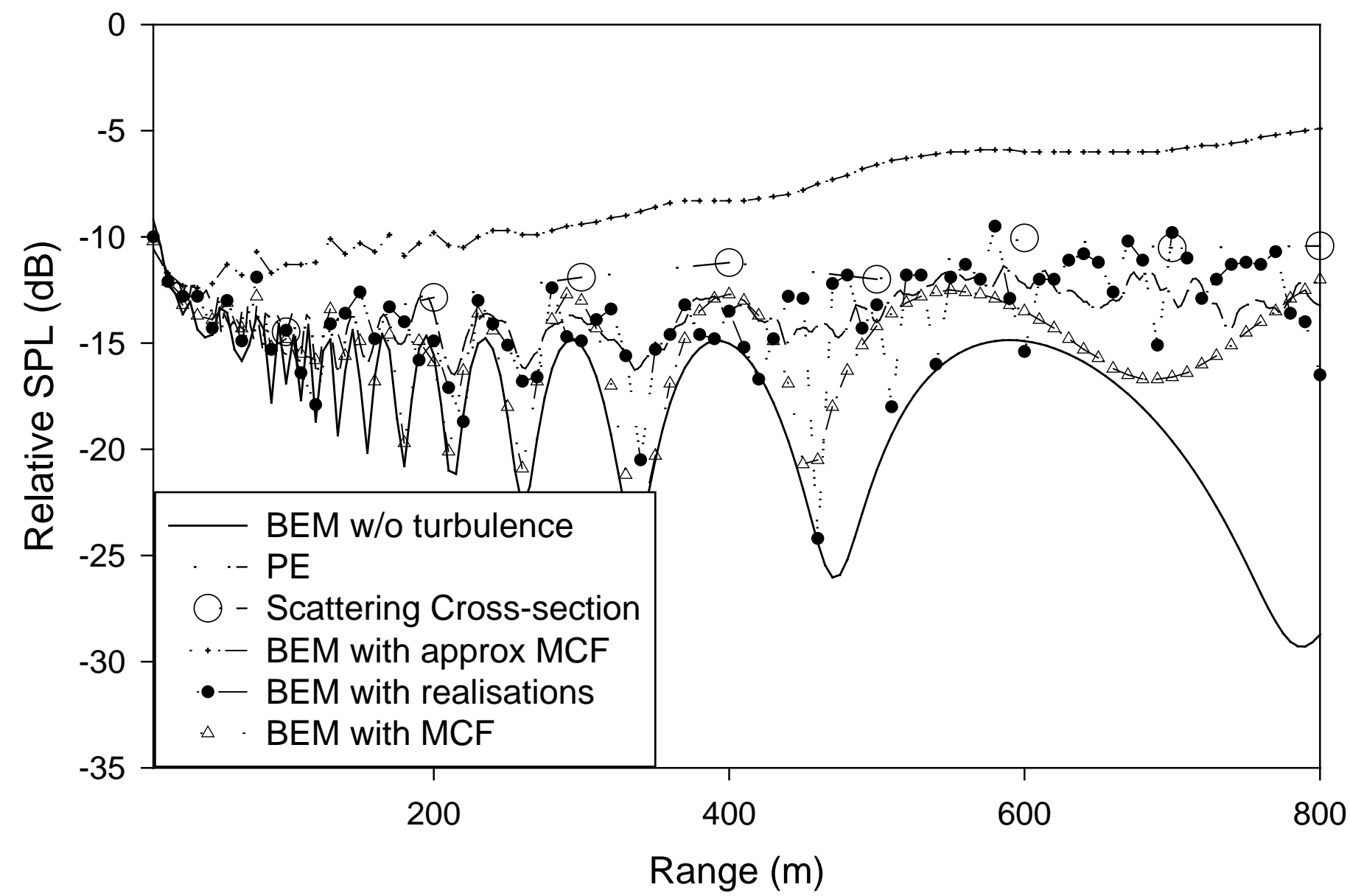

Figure 10 Calculated relative SPL at $500 \mathrm{~Hz}$ as a function of horizontal range from a $20 \mathrm{~m}$ high thin barrier on a hard ground. The source is on the ground $200 \mathrm{~m}$ away from the barrier. The receiver height is the same as the barrier height. $\mu_{o}=3 \times 10^{-6}$ and $\ell=1.1 \mathrm{~m}$. 


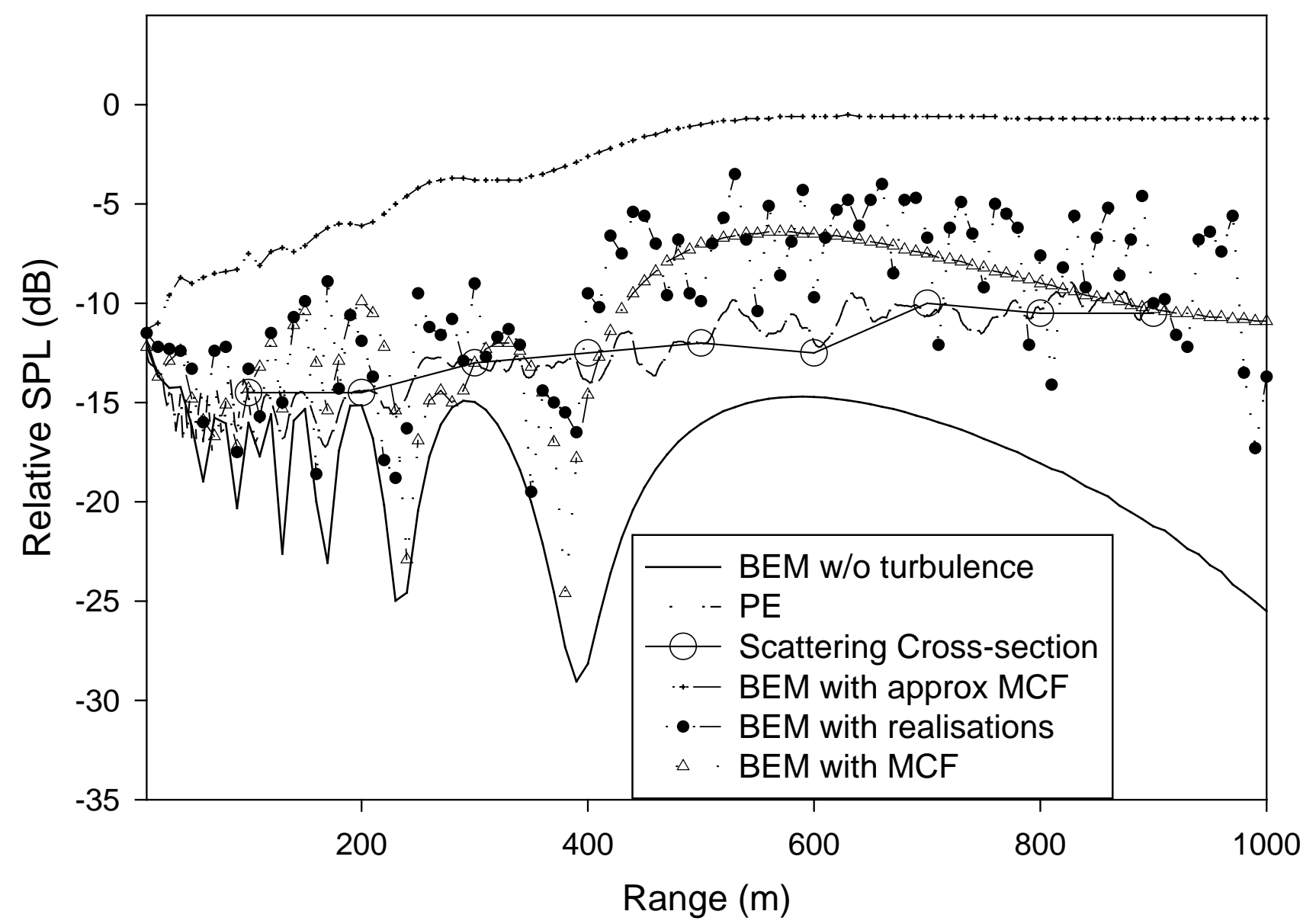

Figure 11 Calculated relative SPL at $1 \mathrm{kHz}$ as a function of horizontal range from a $10 \mathrm{~m}$ high thin barrier on a hard ground. The source is on the ground $100 \mathrm{~m}$ away from the barrier. The receiver height is the same as the barrier height. $\mu_{o}=3 \times 10^{-6}$ and $\ell=1.1 \mathrm{~m}$. 